\title{
Del derecho a la identidad al derecho a las identidades. Un acercamiento conceptual al género y el desarrollo temprano en la infancia
}

\author{
RAÚL MERCER ${ }^{1}$, DALIA SZULIK², MARÍA CARLOTA RAMÍREZ ${ }^{3}$, HELIA MOLINA ${ }^{4}$ \\ 1. Programa de Ciencias Sociales y Salud y PRIGEPP-FLACSO (Facultad Latinoamericana de Ciencias Sociales), Argentina. \\ Proyecto Red de Centros Perinatales del AMBA, Buenos Aires. Argentina. \\ 2. Investigadora del Consejo Nacional de Investigaciones Científicas y Técnicas (CONICET), con sede en FLACSO, Argentina. \\ 3. Programa Regional de Investigación en Género y Políticas Públicas (PRIGEPP) de FLACSO, Argentina. \\ 4. Jefa de la División de Políticas Saludables y Promoción del Ministerio de Salud de Chile. Profesora del Departamento de
} Salud Pública de la Pontificia Universidad Católica de Chile.

Palabras clave: Identidad, Género, desarrollo, diversidad, igualdad.

\section{Introducción}

El conocimiento sobre la conformación de la identidad de género como parte del proceso de desarrollo de la niñez ha sido abordado generalmente desde la psicología evolutiva. Este análisis, destacando los aspectos de individuación, poco ha trascendido al marco poblacional en donde la relación entre género e infancia ha sido poco relevante en los programas y las políticas de salud infantil. Además, la incorporación de la perspectiva de género, ha sido generalmente identificada como una temática inherente a la población adolescente o adulta. Esta situación ha llevado a invisibilizar aquellos aspectos propios del género como determinante de la salud y el desarrollo temprano ${ }^{1}$.

La comunidad científica ha demostrado un interés creciente por la temática atendiendo a reconocer que la construcción de estereotipos, las formas de ser, hacer y relacionarse, tienen sus correlatos con los primeros años de la vida.
Integrar la perspectiva de género al campo de la pediatría implica la necesidad de incorporar un aspecto significativo en el campo profesional no sólo en el abordaje de niños y niñas en la práctica asistencial, sino además, en el mayor conocimiento del rol de las familias, los modos del desarrollo vincular y la internalización de nuevas competencias para la práctica profesional.

El enfoque de género en la niñez es pertinente por varias razones: la construcción de los roles de género que acompañan al desarrollo de los niños y niñas es parte de su socialización. Los y las profesionales de la salud solemos potenciar los estereotipos de género en la familia y podemos, en cambio, jugar un importante papel en su transformación siempre y cuando tengamos los conocimientos, habilidades y actitudes adecuados para aplicar el enfoque de género en nuestra práctica profesional.

El género se constituye como una de las relaciones estructurantes que sitúan al individuo en el mundo y determina a lo largo de su vida, oportunidades, elecciones, trayectorias, vivencias, lugares e intereses. En realidad, las diferencias de género son construcciones sociales que acontecen a lo largo de la vida y que

Correspondencia a:

Raúl Mercer

E-mail: raulmercer@gmail.com 
pueden ser cambiadas, hecho que no acontece con las diferencias biológicas ${ }^{2}$.

La Convención Internacional por los Derechos del Niño $(\mathrm{CIDN})^{3}$ en su Artículo 28 hace referencia al derecho a la igualdad y la no discriminación, entendiendo que cualquier actitud o comportamiento discriminatorio afecta las libertades y posibilidades de desarrollo inclusivo.

El presente artículo tiene como objetivo, abrir un espacio de conocimiento y reflexión que indaga sobre la importancia del género como determinante significativo del desarrollo infantil. Se abordarán aspectos conceptuales, se analizarán las implicancias del género sobre el desarrollo temprano, se indagarán sobre los espacios significativos inherentes a la infancia en donde el género va modelando aspectos del desarrollo y, finalmente, se destacarán algunas ideas y campos de intervención en la materia. El fin último es motivar a la comunidad profesional para promover un acercamiento al tema para que los/as profesionales promuevan una mayor equidad desde los inicios de la vida.

\section{La perspectiva de género en la niñez: Claves para el desarrollo}

El término género se utiliza para describir las características de hombres y mujeres que están basadas en factores sociales, mientras que sexo se refiere a las características que vienen determinadas biológicamente. Las personas nacen con sexo masculino o femenino, pero aprenden a ser niños y niñas que se convierten en hombres y mujeres.

Nuestro conocimiento de la identidad masculina o femenina no se reduce única y exclusivamente a la consideración de una serie de atributos naturales, sino que en gran parte se sustenta en producciones culturales y sociales. En términos biológicos, lo que distingue a las mujeres de los hombres son las diferencias en sus sistemas reproductivos. Sin embargo, esto constituye sólo una parte del complejo conjunto de criterios por los cuales nosotros aprendemos a distinguir la feminidad de la masculinidad. De la misma importancia son las características que las diferentes culturas asignan a aquellos individuos definidos como femeninos y aquellos definidos como masculinos, es decir el género.
Este comportamiento aprendido compone la identidad de género y determina los roles de los géneros. El género asignado y/o asumido afecta la manera como participaremos en el reparto de poder, así como la influencia que tendremos en el proceso de toma de decisiones en todos los ámbitos de la sociedad y particularmente aquellos relacionados con la salud ${ }^{4}$. Los roles de género, o sea, las funciones que se espera desempeñen las niñas y los niños a lo largo de sus trayectos vitales en una sociedad, difieren a través de las culturas y cambian con el tiempo. Del mismo modo, las diferencias entre los géneros y las inequidades en las relaciones de género se expresan a veces en formas disímiles.

\section{¿Somos iguales?}

Las sociedades crecen, se transforman y se enriquecen en su diversidad. Este fenómeno se presenta en los modos de expresarse, de pensar, de ver y sentir. Aceptar y reconocer la diversidad como un atributo valioso y necesario posibilita que las personas puedan desarrollarse y realizarse libremente ${ }^{5}$. Dicho en otros términos: ser más saludables y felices.

Reconocer la diversidad contribuye a la generación de sociedades más cohesivas, menos violentas y tolerantes. Cuando las diferencias generan situaciones de estigmatización, rechazo, discriminación y exclusión, nos encontramos frente a distorsiones en las pautas de convivencia que lamentablemente expresan comportamientos negativos basados, muchas veces, en prejuicios y preconceptos (por ej. homofobia, la xenofobia, etc). Algunas de estas situaciones están arraigadas a tradiciones y preceptos de clase. El presente material se orienta a indagar sobre cómo la dimensión de género está presente desde las etapas iniciales de la vida de las personas y puede condicionar la calidad de las trayectorias vitales. Como se observa en la figura 1, el desarrollo inclusivo se apoya en la visión de derechos y contempla todas y cada una de las dimensiones que hacen a la diversidad en una sociedad.

\section{Comprendiendo las diferencias}

La existencia de diferencias individuales y grupales en una población puede ser expresión 


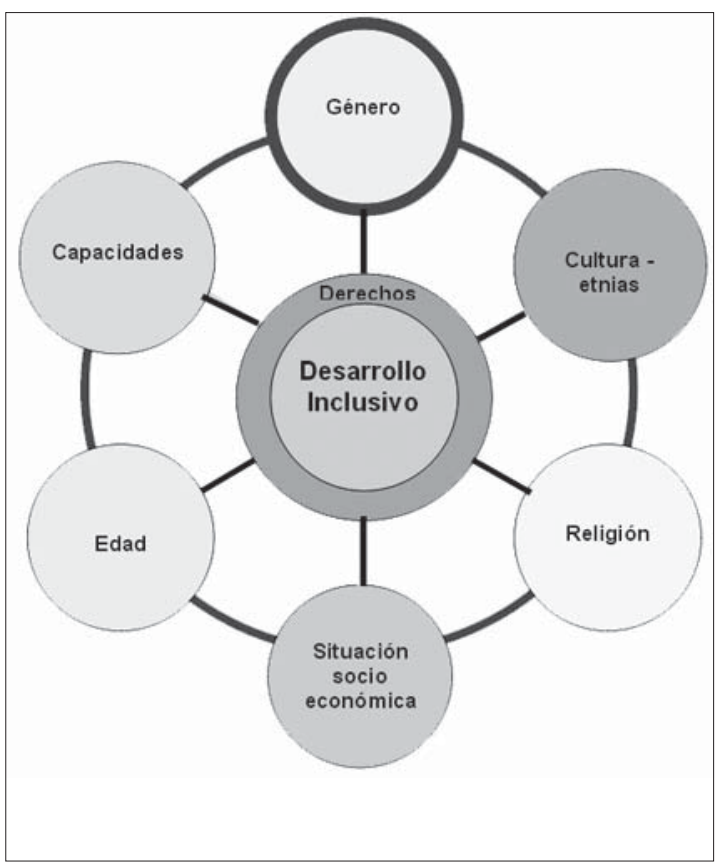

Figura 1. "La flor de la diversidad" (Modelo Raúl Mercer). de múltiples factores y determinantes. Estas diferencias, a su vez, pueden ser explícitas o no, dependiendo del grado de visibilidad que adquieran en un contexto dado. Las diferencias proveen matices, singularidades, y atributos de un conjunto complejo de personas inmersas en procesos continuos de transición. Las siguientes (tabla 1), son algunas de las formas por las cuales las diferencias en la niñez se expresan y su significación en términos de su legitimación social.

\section{La identidad de género como componente del desarrollo temprano}

La construcción de los estereotipos de género es un proceso por el cual se conforman los roles, la conexión entre el género de las personas y sus preferencias y valoraciones sociales. La conformación de los roles de género

Tabla 1. Comprendiendo las diferencias: tipos de diferencias observables en la niñez

\begin{tabular}{|c|c|}
\hline Tipos de diferencias & Comentarios \\
\hline - Diferencias biológicas & $\begin{array}{l}\text { Son las que acompañan al ciclo de vida y que hace que cada estadio del } \\
\text { crecimiento y desarrollo sea diferente. También son aquellas que están deter- } \\
\text { minadas por patrones constitucionales, generalmente genéticos y que expre- } \\
\text { san variaciones en las características del crecimiento y desarrollo. Por ejemplo, } \\
\text { las niñas se desarrollan antes que los varones. A su vez, los varones poseen, } \\
\text { en general, mayor desarrollo y fuerza muscular que las mujeres. Estas diferen- } \\
\text { cias, son aceptadas socialmente. }\end{array}$ \\
\hline - Complejas (basadas en tendencias seculares) & $\begin{array}{l}\text { La tendencia secular del crecimiento pone de manifiesto las cualidades de las } \\
\text { poblaciones para expresar su potencialidad biológica para crecer, hecho que, } \\
\text { por ejemplo, se observa en aquellos países con un alto grado de desarrollo } \\
\text { socioeconómico. }\end{array}$ \\
\hline - Variabilidad normal & $\begin{array}{l}\text { Es aquella que responde a los procesos de individuación y que le confiere } \\
\text { una característica peculiar a cada persona. Es lo que hace que seamos dife- } \\
\text { rentes unos/as de otros/as. Es un fenómeno aceptado socialmente y que } \\
\text { contribuye a nuestra riqueza como colectivo social. }\end{array}$ \\
\hline - Diversidad de valores & $\begin{array}{l}\text { Es la que responde a patrones de desarrollo cultural y transmisión de valores } \\
\text { de credo y de tradiciones. Aceptada como un valor legítimo de diversidad } \\
\text { aunque en ciertos grupos puede condicionar situaciones de estigmatización } \\
\text { y conflicto. }\end{array}$ \\
\hline - Asociadas a problemas de salud & $\begin{array}{l}\text { Ciertas afecciones pueden condicionar alteraciones del desarrollo físico o } \\
\text { intelectual y del estado de salud en general (generalmente asociadas a } \\
\text { discapacidades intelectuales, sensoriales, motrices). }\end{array}$ \\
\hline - Inequidades & $\begin{array}{l}\text { Son aquellas diferencias existentes en una sociedad debidas a la presencia de } \\
\text { patrones asimétricos en la distribución del poder y en la posibilidad de ejercer } \\
\text { derechos básicos. Su existencia afecta la salud y el desarrollo de las personas } \\
\text { producto de situaciones de discriminación ya sea por su edad, cultura, reli- } \\
\text { gión, orientación sexual, estado de salud, etc. Expresan situaciones de injus- } \\
\text { ticia social. }\end{array}$ \\
\hline
\end{tabular}


comienza durante los primeros años de la vida. Existen dos perspectivas que explican cómo la identidad de género es formada en la niñez temprana, con evidencia suficiente que indica que el proceso de socialización tiene un rol preponderante.

- Teoría sobre roles sexuales: Básicamente los niños y niñas aprenden la forma de relacionarse con el mundo a través de observar cómo actúan quienes los/as rodean, por la forma en como son reconocidos/as o castigados/as después de un comportamiento determinado. De esta manera, niños y niñas modelan sus conductas sobre el comportamiento de familiares, amigos/as e imágenes del mismo sexo que se le cruzan en el día a día.

- Teoría de género relacional: Considera a los niños y niñas activamente involucrados/ as en desarrollar su propia identidad y argumenta en contra del enfoque que dice que todos los niños y niñas tienen intereses y comportamientos similares. El concepto de género para los niños y niñas cambia en forma constante, dependiendo del contexto y de determinantes como la clase social, la etnia, la religión, la edad y la cultura.

Alrededor de los dos años, los niños y niñas comienzan a nombrar correctamente su sexo y el de otras personas. Una vez que estas categorías básicas de género están establecidas, empiezan a clasificar las actividades y las conductas y asimilan una gran amplitud de estereotipos de género. Los niños y niñas pequeños asocian con el sexo muchos artículos de ropa, herramientas, elementos del hogar, juegos, ocupaciones y comportamientos. Sus acciones van de acuerdo a esta idea. En el período preescolar, los estereotipos de género de los pequeños se fortalecen y parecen operar con reglas rígidas y no con normas flexibles. En la conformación de los estereotipos de género participan influencias:

- Genéticas: Las diferencias de comportamientos entre niños y niñas parecen visibles en muchas culturas. Esto nos lleva a considerar las posibles influencias genéticas. Durante el período preescolar, las niñas aumentan la búsqueda de otras niñas y disfru- tan jugando con sus compañeras. Los niños parecen preferir actividades grupales de niños como correr, escalar y jugar a pelear. Existe un amplio rango de factores ambientales que construyen e interaccionan con influencias hereditarias el conocimiento del género y de los roles en los niños y niñas.

- Relacionales: La familia y los pares son modelos importantes en la comunidad. El ambiente social juega un papel importante en el desarrollo de los estereotipos de género durante la infancia temprana. Cuando se habla de los valores de crianza, los padres tienden a hablar del rendimiento, competencia y el control de emociones como elementos importantes para los hijos, mientras que el cariño y las características de las niñas son importantes para las hijas.

Estas prácticas y expectativas influencian las acciones de los padres y las madres. Los padres y las madres compran juguetes que promueven comportamientos que refuerzan los diferentes estilos de juego. A través de sus respuestas, refuerzan conductas estereotípicas de género. Ha sido sugerido que los padres se comprometen en conductas más discriminatorias que las madres. Ellos motivan más las conductas apropiadas de género y presionan más a los hijos que a las hijas.

Los niños y niñas del mismo sexo refuerzan de forma importante las creencias estereotípicas y los comportamientos. Alrededor de la edad de tres años, los compañeros del mismo sexo refuerzan de forma positiva los juegos tipificados de género, alabando, imitando o disfrutando actividades con compañeros de la misma edad. De forma similar, los preescolares son criticados por sus compañeros cuando seleccionan actividades del sexo opuesto. Los niños también desarrollan diferentes estilos de interacciones sociales en grupos segregados de compañeros.

\section{Espacios sociales y desarrollo temprano}

Las posibilidades de desarrollo pleno están íntimamente vinculadas con la calidad de experiencias tempranas que niños y niñas atraviesan en los diferentes ámbitos y organizadores que 
van moldeando sus potencialidades y personalidades.

Es importante entender que en todos los espacios sociales por los cuales transcurre la vida de un niño/a y adolescente (instituciones educativas, servicios de salud, familias, barrios, otras) se puede establecer la existencia de un "orden de género". Este orden es puesto en escena por la dinámica de tres factores principales ${ }^{7}$ :

a) Estructuras: Abarcan las reglas, formal o informalmente establecidas, que definen los alcances y posibilidades de la experiencia y de los patrones que las limitan.

b) Prácticas: Son los comportamientos cotidianos y los procesos que le dan vida y sentido sustantivo a la estructura y que a su vez la reproducen.

c) Las y los agentes: Las personas que actúan e interactúan y que a su vez llevan a las prácticas y al contexto institucional en alguna medida, sus propias variantes personales.

\section{Los contextos familiares}

La infancia y la familia son nociones estrechamente relacionadas entre si ya que es difícil considerar una de ellas independientemente de la otra. En tanto esta última constituye un fenómeno universal, existe una tendencia a naturalizar las relaciones que se establecen en su interior identificando la unidad biológica de reproducción con la red de relaciones sociales que la conforman.

Inscripta en el espacio privado, la familia representa la puerta de entrada del sujeto al mundo social y es un referente fundamental en tanto da origen a la identidad individual y social. Sujeta al ámbito de lo privado, por oposición a la esfera pública, la organización familiar reproduce en mayor o menor medida las reglas sociales vigentes en un momento histórico determinado como así también las relaciones de género predominantes. Es en el ámbito de la familia donde primariamente y a través del lenguaje, se construye una imagen del mundo exterior, y se aprende a ordenar y dar sentido a la experiencia vivida.

Los cambios científicos y sociales ocurridos en las últimas décadas promovieron una alteración de los principios en los que se apoyaban las relaciones tradicionales de complementariedad de roles y jerarquías, por otras formas de organización familiar en las que se privilegia la afirmación de la individualidad.

El descubrimiento de la píldora anticonceptiva en los años 60 y la incorporación masiva de la mujer al mercado de trabajo dieron inicio a un proceso de transformación que permitió independizar a la mujer de los roles de madre y esposa ${ }^{8}$. Los avances científicos y tecnológicos registrados en la década de los 80 resultaron a su vez en intervenciones sobre la reproducción humana introduciendo la dimensión de "elección" contrapuesta a la de "destino" históricamente asociada al universo naturalizado de la familia. Más recientemente, los aportes de la biología molecular produjeron nuevas tensiones acerca del lugar que el hombre ocupa en la familia como padre y también en el ejercicio de la sexualidad masculina y la actitud que el varón asume frente a la concepción que fue considerada hasta entonces como un problema fundamentalmente "femenino".

Estas transiciones en las familias y, en las relaciones de género se expresan de manera diversa según los distintos sectores sociales dentro de una sociedad divida en clases y caracterizada por un acceso desigual a los recursos. El aumento de familias con jefatura femenina, las familias ampliadas por múltiples casamientos de los padres, las familias transnacionales producto de la feminización de la migración internacional, y las constituidas por progenitores padres y madres del mismo sexo son expresión de los cambios que han contribuido a desnaturalizar a "la familia" como orden natural.

En el contexto actual, los roles de género, los derechos y deberes entre padres/madres e hijos/as, como también el ejercicio de autoridad, las concepciones acerca de la sexualidad y los modos de ejercer la maternidad y la paternidad, se construyen a partir de constantes negociaciones a través de las cuales son a su vez revisados y redefinidos en un proceso permanente de construcción que da cuenta de la diversidad de las "familias" en oposición a un modelo único e ideal de familia.

\section{Pautas de crianza y relaciones de género}

Las pautas diferenciales de crianza se manifiestan en una división sexual que conlleva 
una distribución diferente de las tareas, responsabilidades y recursos para varones y niñas y encubre desigualdades en la distribución del poder: A las niñas y adolescentes se les asignan determinadas tareas, preferentemente circunscriptas a la esfera doméstica (alimentación de hermanos/as, cuidado de adultos) y de los varones se espera que sean los principales proveedores del hogar, proyectando su formación hacia la esfera pública. Se podría objetar que nadie impide a las mujeres o a los varones ser diferentes y cumplir otras funciones. Pero salir del molde asignado para cada uno/a acarrea sanciones sociales. Así, las influencias de género, como las de clase social, al configurar contextos de interacción específicos, repercuten de forma sustancial en los procesos de desarrollo y construcción de identidad de cada niño/a o adolescente.

Las relaciones que se establecen entre los géneros remiten a redes de significado presentes en diversas dimensiones de la vida de cada persona. Este proceso conforma la denominada socialización de género. Considerando el proceso de socialización como permanente y siempre inconcluso, las categorías de género son constantemente reconstruidas por las personas desde su infancia, en sus interacciones, valores, roles y normas. Desde esta perspectiva, las relaciones de género atraviesan todo el tejido social de manera dinámica, manifestándose de diferente forma en cada grupo social ${ }^{9}$.

Es en los espacios de convivencia cotidiana, más particularmente en la familia, en donde los niños y niñas aprenden e internalizan su género, de acuerdo a las exigencias de su contexto local. El cotidiano familiar está fuertemente influenciado por la organización de género que trasciende el espacio doméstico. La jerarquización de género se presenta en rutinas, juegos y proyectos. En las relaciones de género, las jerarquías son creadas, reproducidas y mantenidas por la interacción entre los miembros del hogar. Por lo tanto, la familia es la institución primaria para la organización de las relaciones de género en la sociedad, donde se puede ejercer los derechos individuales, pero también donde se establecen relaciones de poder desigual y asimétrico, se presenta el conflicto social, la discriminación y el maltrato, siendo los más débiles, muchas veces, las mujeres, niños y niñas y ancianos/as.

Las divisiones de género modulan las vidas de niños, niñas y adolescentes de manera fundamental en el desarrollo de su identidad y sus capacidades futuras de socialización. Como individuos con identidades particulares y como actores en una infinita variedad de contextos sociales, son moldeados por su feminidad y su masculinidad. En un sentido u otro los niños y niñas son limitados de acuerdo a su pertenencia a un grupo particular de género. En la mayoría de las sociedades estas variaciones son utilizadas para justificar más inequidades que afectarán en mayor proporción al género femenino que al masculino con implicancias negativas sobre la salud y el desarrollo.

Otros elementos sociales que influencian en este proceso son los medios de información y comunicación, los juegos, los programas de televisión, los libros o los materiales educativos, que pueden reforzar ciertos estereotipos, por ejemplo, contribuyendo a invisibilizar a las mujeres en el espacio público o presentarlas por fuera de las profesiones consideradas tradicionalmente masculinas, o utilizando herramientas potentes, como el lenguaje sexista o las imágenes en la publicidad de juguetes y actividades de ocio (donde las niñas se relacionan con actividades domésticas y colores pastel, y los niños con las más agresivas y de riesgo como las armas o los vehículos de motor).

\section{Los espacios institucionales \\ (las escuelas, los servicios de salud)}

Las marcas identitarias de los géneros están fuertemente determinadas por el universo de relaciones que se sostienen a lo largo de los diversos cursos vitales y que a su vez forman parte de las estructuras de las instituciones. Se puede afirmar, entonces, que las conductas de género son constituidas y al mismo tiempo son constituyentes de las instituciones y organizaciones a que ellas dan lugar. Las reglas de juego que definen las relaciones entre los géneros están presentes en la universalidad de los contextos normativos y en las prácticas organizacionales derivadas de ellas.

Un escenario en el que es posible reconocer claramente estos factores es el de las institu- 
ciones escolares que funcionan dentro de un marco definido de pautas y reglas formales que definen su estructura, la cual es permanentemente modificada y reformulada por la vida cotidiana, las interacciones sociales y los aportes de cada uno de los actores presentes.

Los maestros y maestras motivan a los niños y niñas a conformar roles de género. La segregación sexual y las respuestas estereotípicas son muy comunes. Durante la edad preescolar, las niñas son motivadas a participar en actividades estructuradas de adultos. Se pueden ver con frecuencia acompañando a la maestra y siguiendo órdenes. Los ambientes de la vida diaria de los niños contienen muchos ejemplos de conductas de roles de género tradicionales que ejercen una influencia poderosa en el proceso de desarrollo del género. Los niños y niñas pequeños no sólo imitan de forma pasiva las respuestas conectadas con el género. Por el contrario, son observadores detallistas de los ambientes circundantes y desarrollan un proceso de preferencias de género que inhibe sus propios intereses y capacidades.

Otro escenario es el de los servicios de salud. Reconocer la importancia de aplicar el enfoque de género en este ámbito resulta en una mejor calidad de atención, planificar acciones que sean más efectivas y que, al mismo tiempo, promuevan igualdad y equidad desde la niñez.

Como plantea Reyes Zapata, el enfoque de género nos da elementos para entender mejor el proceso de salud y enfermedad. Es posible acceder mediante el enfoque de género al menos a tres niveles de análisis ${ }^{10}$ :

1) Las diferentes maneras en que niños, niñas $\mathrm{y}$ adolescentes tienen problemas de salud asociados a sus roles de género.

2) La manera distinta en que se proveen servicios a niños, niñas y adolescentes, citada por algunos autores como "disparidades de género".

3) La manera en que las proveedoras y los proveedores de servicios debieran considerar los roles de género y su impacto sobre la salud para dar una mejor atención.

Es evidente que incorporar el enfoque de género en la atención y cuidado de la salud permitiría otorgar servicios de mayor calidad, ya que contextualiza a las usuarias y usuarios como sujetos de derecho más allá de los límites biológicos que, de manera frecuente, circunscriben la atención médica.

\section{Los campos de intervención}

Al pensar en aplicar el enfoque de género en el terreno operativo deben considerarse aquellos factores que expresan situaciones de inequidad de género sobre la salud y el desarrollo de niños, niñas y adolescentes:

- Diferencias en los factores de riesgo y de protección de la salud.

- Desequilibrio entre las necesidades en salud y el acceso a recursos.

- Diferencias sobre el modo a través del cual las políticas y programas afectan a las niñas con respecto a los niños y viceversa.

\section{Los servicios de salud}

Los servicios de salud (la consulta ambulatoria y la atención hospitalaria) conforman espacios para reorientar y adoptar prácticas basadas en derechos, en nuestro caso, reconociendo la dimensión de género. Como todo campo innovador, induce a un esfuerzo exploratorio, la investigación y la generación de evidencias que apoyen las prácticas en los servicios de salud. Algunas de las acciones propuestas podrían ser las siguientes:

- Promover el logro exitoso de la lactancia exclusiva, considerando el grado en que las necesidades de las mujeres (privacidad, trabajo y otras) y las diferencias culturales son tomadas en cuenta, respetando las decisiones que atiendan los derechos de los recién nacidos al acceso a la mejor calidad de alimentación y cuidados, como el de sus madres a proveerlos.

- Promover y alentar el involucramiento de la pareja y la familia en los cuidados en el hogar.

- Considerar y destacar la participación del padre en la crianza.

- Detectar posibles diferencias en la valorización de la salud de los niños con respecto a de las niñas, así como la exposición diferencial a riesgos entre niños y niñas.

- Estimular a través del juego, la comunicación y la interacción social, tomando en cuen- 
ta el acceso diferencial a las actividades recreativas y el desarrollo de estereotipos de género a través de la actividad lúdica.

- Analizar los hábitos alimentarios y si es que existen diferencias entre niñas y niños en las familias.

- Distinguir diferencias de género en la detección y tratamiento de la depresión, problemas de aprendizaje y de comportamiento.

- Indagar en comportamientos posiblemente condicionados por normas y valores de género que puedan influir en modos de ser saludable o no dentro de las familias.

- Promover formas de relacionamiento entre niños y niñas que no sean violentas o que acepten los comportamientos agresivos como un elemento constitutivo necesario de los varones.

- Reconocer las diferencias en los patrones inicio de comportamientos de riesgo de niños, niñas y adolescentes (incluyendo las diferencias en el consumo de sustancias como alcohol, tabaco y drogas).

\section{Los programas de salud infantil y adolescencia}

Estas cuestiones resultan fundamentales al momento de planificar e implementar programas de salud destinados a niños/as y adolescentes incorporando la perspectiva de género, lo que se evidencia en la reciente iniciativa de la OMS Europea ${ }^{11}$. Recordemos que los modelos de abordaje de los programas de salud infantil, perpetúan estereotipos de género en lugar de generar una visión integradora para hombres y mujeres puedan expresar sus diferentes roles en las familias y la sociedad ${ }^{12}$.

\section{La formación de recursos humanos}

Un intento innovador por indagar la dimensión de género lo constituye el Seminario de Infancias y Género. Se trata de una actividad formativa que se desarrolla en el marco del Programa Regional de Investigación en Género y Políticas Públicas (PRIGEPP) de FLACSO, Argentina. Esta iniciativa intenta cubrir un vacío en materia de la temática de género desde las etapas tempranas de la vida y por otra parte trata de satisfacer una demanda de profesionales de la Región que permita nuevas lecturas hacia la problemática de la niñez. A su vez se propone construir un espacio innovador para la promoción de cambios en las prácticas profesionales que contribuyan a la reorientación de los servicios, la docencia, investigación, asistencia y gestión de programas hacia el enfoque de equidad de género en la infancia. Para ello, a lo largo del seminario se analizan las realidades de las familias y de la niñez de la región en contextos de cambio, incorporando dimensiones no tradicionales en relación con las familias y con la niñez (enfoque de ciclo de vida, determinantes de salud y calidad de vida, desarrollo integral, e inclusión de la niñez con énfasis en la dimensión de género). Finalmente, se identifican y analizan las problemáticas emergentes que afectan a la infancia y las familias y las respuestas sociales (políticas, profesionales y comunitarias) existentes para abordarlas.

Por su parte, Chile integra junto a Argentina, Colombia y Uruguay la Iniciativa del Cono Sur sobre Salud y Derechos de la Niñez ${ }^{13}$. Esta iniciativa, de carácter multisectorial tuvo sus orígenes a partir de una currícula elaborada por la Academia Americana de Pediatría y el Royal College of Paediatrics (UK). Promueve la integración de la perspectiva de derechos en los servicios de salud para niños y niñas en el marco de la CIDN. Para ello, se cuenta con una propuesta de formación destinada a integrantes del equipo de salud, hoy en fase de implementación. Durante la convocatoria realizada en Santiago para Taller de Formación de Formadores de los cuatro países del Cono Sur, se contó además con la participación de la Sociedad Chilena de Pediatría, OPS, UNICEF, la Pontificia Universidad Católica, MINSAL y MIDEPLAN. Por su parte, la Universidad de Chile, incorporó el desarrollo temprano y el género como parte de la currícula de sus actividades de post-grado, esta vez, en el marco de la formulación de proyectos, programas y políticas de salud ${ }^{14}$.

\section{Difusión y abogacía}

La incorporación del enfoque de género como un componente del desarrollo temprano ha sido considerada en los contenidos de algunos textos y publicaciones destinados a los integrantes del equipo de salud ${ }^{15}$. Durante los años recien- 
tes, una serie de eventos realizados en Chile y Argentina, contemplaron las perspectivas de género y desarrollo como ejes de debate en el ámbito de la Salud Pública ${ }^{16-19}$.

\section{Comentarios finales}

En este artículo nos propusimos plantear y analizar la necesidad de incluir la perspectiva de género en la infancia, destacando la importancia de las intervenciones tempranas para llegar a la vida adulta con mayor equidad. Este resultado es parte de un proceso que permitiría alcanzar una expansión de los derechos ciudadanos, incluyendo cuestiones que tradicionalmente fueron excluidas de estos derechos, como ser la sexualidad y el cuidado infantil ${ }^{20}$. A partir de las argumentaciones expuestas, existen evidencias suficientes que ameritan abordar al género desde etapas tempranas de la vida, incluso antes de la concepción del nuevo ser, desde el momento que cada pareja "negocia democráticamente" el mejor momento para asumir su paternidad y maternidad, hecho que, lamentablemente no acontece en nuestra región. Por otra parte, la conformación de estereotipos de género en los primeros años de la vida, va conformando modos de relacionamiento asimétricos de poder que se irán consolidando a lo largo del ciclo vital a partir de los mandatos familiares e institucionales. Quienes trabajamos en salud, ya sea desde las políticas, programas y provisión de servicios, estamos apelados a considerar la dimensión de género como un determinante social que afecta la salud y calidad de vida de las personas. Esta perspectiva nos permitirá pensar nuevas modalidades de intervención que promuevan un desarrollo temprano basado en los derechos de la niñez y no exclusivamente en los mandatos de los adultos.

\section{Referencias}

1.- Early Child Development: a Potent Equalizer. Final Report of the Knowledge Network on Early Child Development. Commission of Social Determinants of Health. WHO, 2007.

2.- Chiarotti S: Aportes al derecho desde la teoría de género. http://www.insgenar.org.ar/documentos/Aportesal-Derecho-desde-la-teoria-de-Genero.pdf

3.- Convención Internacional sobre los Derechos del Niño,
Asamblea General de las Naciones Unidas, 20 de noviembre de 1989.

4.- Unequal, Unfair, Ineffective and Inefficient. Gender Inequity in Health: Why it exists and how we can change it. Final Report to the WHO Commission on Social Determinants of Health September. 2007. Women and Gender Equity Knowledge Network.

5.- Sen A: Desarrollo y Libertad. 2000. Editorial Planeta, Argentina.

6.- Fatherhood and Health outcomes in Health. http:// www.euro.who.int/document/e91129.pdf Europe World Health Organization 2007.

7.- García Prince E: Hacia la institucionalización del enfoque de género en políticas públicas. Documento elaborado para Fundación Friedrich Ebert, Caracas, 2003.

8.- Jelin E: Pan y Afectos. La Transformación de las Familias. 1998 Fondo de Cultura Económica.

9.- Vielma J: Estilos de crianza, Estilos educativos y Socialización: ¿Fuentes de Bienestar Psicológico? Acción Pedagógica, Vol 12, $\mathrm{N}^{\circ} 1,2003$.

10.- Reyes-Zapata $H$, et al: Un sistema de medición de la calidad de los servicios de salud sexual y reproductiva desde una perspectiva de género, Documento de trabajo núm 29, Population Council, 1999.

11.- WHO: European Strategy for child and adolescent health and development. Gender tool. WHO/ Europe. WHO, 2007. www.euro.who.int/document/gem/ eurostrat_gender_tool.pdf

12.- Mercer R, Karolinski A: La salud materno infantil: momento de cuestionamientos. ¿Cambio de paradigma? 2007. Editorial. Revista Hospital Durand, Argentina. (3): 84-6.

13.- Iniciativa sobre Salud y Derechos del Niño del Cono Sur. FLACSO/CRED-PRO: Con la participación de equipos profesionales e instituciones de Argentina, Chile, Colombia y Uruguay.

14.- X Escuela Internacional de Verano: Escuela de Salud Pública de la Universidad de Chile. Curso Desarrollo Integral de la Primera Infancia. Desafíos para la Salud Pública. 14 al 18 de enero de 2008.

15.- Landers C, Mercer R, Molina H, Young M: Desarrollo Integral en la Infancia: Una Prioridad para la Salud. Manual de Desarrollo Temprano para Profesionales de la Salud. 2006. Organización Panamericana de la Salud, Banco Mundial, J\&J Pediatric Institute.

16.- MINSAL: III Congreso Chileno de Promoción de la Salud. Centro de Convenciones de los Almendros. 1012 de enero de 2007.

17.- MINSAL-OPS: Taller de Desarrollo Integral en la Infancia. Santiago de Chile, 10 y 11 de mayo de 2007.

18.- Mercer R: I Congreso Chileno de Salud Pública. La Convención de los Derechos del Niño y la Protección Integral de la Infancia. Santiago de Chile, 18-20 de julio de 2007.

19.- Sociedad Argentina de Pediatría: X y XI Congreso Argentino de Pediatría Social $(2005,2008)$. Buenos Aires, Argentina. www.sap.org.ar

20.- Meer S, Sever C: Género y Ciudadanía. Informe General. Bridge, Developmet Gender. http://www.ids.ac.uk/ bridge. 